\title{
Global Solutions for the Kuramoto-Sivashinsky Equation Posed on Unbounded 3D Grooves
}

\author{
N. A. Larkin \\ Department of Mathematics, State University of Maringá, Pr, AV. Colombo 5790, 87020-900, Maringá, Parana, Brazil \\ E-mail: nlarkine@uem.br
}

Received: 02 August 2021; Revised: 26 September 2021; Accepted: 26 September 2021

\begin{abstract}
Initial boundary value problems for the three-dimensional Kuramoto-Sivashinsky equation posed on unbounded 3D grooves (that may serve as mathematical models for wildfires) were considered. The existence and uniqueness of global strong solutions as well as their exponential decay have been established.
\end{abstract}

Keywords: kuramoto-sivashinsky equation, global solutions, decay in unbounded domains

MSC: 35B35, 35K91, 35Q53

\section{Introduction}

This work concerns the existence and uniqueness of global strong solutions as well as exponential decay rates of solutions to initial boundary value problems for the three-dimensional Kuramoto-Sivashinsky equation (K-S):

$$
\phi_{t}+\Delta^{2} \phi+\Delta \phi+\frac{1}{2}|\nabla \phi|^{2}=0
$$

Here $\Delta$ and $\nabla$ are the Laplacian and the gradient in $\mathbb{R}^{3}$. In [1], Kuramoto studied the turbulent phase waves, and Sivashinsky in [2] obtained an asymptotic equation which modeled the evolution of a disturbed plane flame front. See also [3]. Moreover, K-S equation has applications in the study of dynamics of plasma and flow of liquid films on inclined planes, [4]. In [5-8], mathematical results on initial and initial boundary value problems for one-dimensional (1) are presented, see references there for more information. Multi-dimensional problems for various types of (1) can be found in [9-15] with some results on the existence, regularity and, nonlinear stability of solutions. In [10-13], stability of global solutions for the K-S equation posed on a rectangle with the width $L_{2}$ and the length $L_{1}$, where $L_{2}$ is a decreasing function of $L_{1}$ and initial data depend on $L_{1}$, was studied. This did not allow to study initial boundary value problems posed on strips. Absence of published results on three-dimensional problems, motivated us to study the existence, uniqueness, and stability of global solutions for the three-dimensional K-S equation. Our approach does not include dependence of $L_{2}$ on $L_{1}$. This allows to prove the existence, uniqueness, and exponential decay of global solutions in two and three dimensions making use of the same approach with the unique restriction on the width of a domain. The

Copyright (C2021 N. A. Larkin.

DOI: https://doi.org/10.37256/cm.2420211075

This is an open-access article distributed under a CC BY license

(Creative Commons Attribution 4.0 International License)

https://creativecommons.org/licenses/by/4.0/ 
shortage of this approach is smallness conditions for the initial data.

For three dimensions, (1) can be rewritten in the form of the following system:

$$
\begin{gathered}
\left(u_{j}\right)_{t}+\Delta^{2} u_{j}+\Delta u_{j}+\frac{1}{2} \sum_{i=1}^{3}\left(u_{i}^{2}\right)_{x_{j}}=0 \\
\left(u_{j}\right)_{x_{i}}=\left(u_{i}\right)_{x_{j}}, i \neq j ; i, j=1,2,3
\end{gathered}
$$

where $u_{j}=\phi_{x_{j}}$. The first essential problem that arises while one studies either (1) or (2)-(3) is a destabilizing effect of $\Delta u_{j}$ that may be damped by a dissipative term $\Delta^{2} u_{j}$ provided a domain has some specific properties, see condition (13) of Theorem 3.1. Naturally, the so-called "thin domain" appears where some dimensions are small while the others may be arbitrarily large. The second essential problem is the presence of semi linear interconnected terms in (2). This does not allow to obtain first estimate independent of solutions and leads to a connection between geometric properties of a domain and initial data, see (14) of Theorem 3.1.

Our work has the following structure: Section 1 is Introduction. Section 2 contains notations and auxiliary facts. In Section 3, formulation of an initial boundary value problem for (2)-(3) posed on unbounded grooves is given. The existence and uniqueness of a global strong solution, exponential decay of the $L^{2}$-norm have been established. Section 4 contains ideas of applications results obtained in our paper to some practical problems. Section 5 contains conclusions.

\section{Notations and auxiliary facts}

Let $\Omega$ be a domain in $\mathbb{R}^{3}$ and $x=\left(x_{1}, x_{2}, x_{3}\right) \in \Omega$. We use the standard notations of Sobolev spaces $W^{k, p}, L^{p}$ and $H^{k}$ for functions and the following notations for the norms [16]: for scalar functions $f(x, t)$

$$
\begin{aligned}
& \mathbb{R}^{+}=\left\{t \in \mathbb{R}^{1} ; t>0\right\}, \\
& \|f\|^{2}=\int_{\Omega}|f|^{2} d \Omega, \\
& \|f\|_{L^{p}(\Omega)}^{p}=\int_{\Omega}|f|^{p} d \Omega, \\
& \|f\|_{W^{k, p}(\Omega)}^{p}=\sum_{0 \leq \alpha \leq k}\left\|D^{\alpha} f\right\|_{L^{p}(\Omega)}^{p}, \\
& \|f\|_{H^{k}(\Omega)}=\|f\|_{W^{k, 2}(\Omega)} .
\end{aligned}
$$

When $p=2, W^{k, p}(\Omega)=H^{k}(\Omega)$ is a Hilbert space with the scalar product

$$
((u, v))_{H^{k}(\Omega)}=\sum_{|j| \leq k}\left(D^{j} u, D^{j} v\right),\|u\|_{L^{\infty}(\Omega)}=\operatorname{ess}^{\sup _{\Omega}}|u(x)| .
$$

We use a notation $H_{0}^{k}(\Omega)$ to represent the closure of $C_{0}^{\infty}(\Omega)$, the set of all $C^{\infty}$ functions with compact support in $\Omega$, with respect to the norm of $H^{k}(\Omega)$.

Lemma 2.1 (Steklov's Inequality [17]). Let $v \in H_{0}^{1}(0, L)$. Then 


$$
\frac{\pi^{2}}{L^{2}}\|v\|^{2} \leq\left\|v_{x}\right\|^{2}
$$

Lemma 2.2 (Differential form of the Gronwall Inequality. See [18], Lemma 2.2.). Let $I=\left[t_{0}, t_{1}\right]$. Suppose that functions $a, b: I \rightarrow \mathbb{R}$ are integrable and a function $a(t)$ may be of any sign. Let $u: I \rightarrow \mathbb{R}$ be a differentiable function satisfying

$$
u_{t}(t) \leq a(t) u(t)+b(t), \text { for } t \in I \text { and } u\left(t_{0}\right)=u_{0},
$$

then

$$
u(t) \leq u_{0} e^{\int_{t_{0}}^{t} a(\tau) d \tau}+\int_{t_{0}}^{t} e^{\int_{t_{0}}^{s} a(r) d r} b(s) \cdot d s
$$

The next Lemmas will be used in estimates:

Lemma 2.3 (See [19], Lemma 3.5.). Let $v \in H_{0}^{1}(\Omega)$ and $n=3$, then

$$
\|v\|_{L^{4}(\Omega)} \leq 2^{\frac{1}{2}}\|v\|^{\frac{1}{4}}\|\nabla v\|^{\frac{3}{4}}
$$

\section{K-S system posed on a groove}

Define a groove

$$
D=\left\{x \in \mathbb{R}^{3} ; x_{1} \in \mathbb{R}^{1}, x_{2} \in(0, B),(B>0), x_{3} \in \mathbb{R}^{+}\right\}, Q_{t}=(0, t) \times D .
$$

Lemma 3.1. Let $f \in H_{0}^{2}(D)$. Then

$$
\begin{aligned}
& a\|f\|^{2} \leq\|\nabla f\|^{2}, a^{2}\|f\|^{2} \leq\|\Delta f\|^{2}, a\|\nabla f\|^{2} \leq\|\Delta f\|^{2}, \\
& \text { where } a=\frac{\pi^{2}}{B^{2}} \text {. }
\end{aligned}
$$

Proof. By definition,

$$
\|\nabla f\|^{2}=\sum_{i=1}^{3}\left\|f_{x_{i}}\right\|^{2} .
$$

Making use of Steklov's inequalities, we get

$$
\|\nabla f\|^{2} \geq\left\|f_{x_{2}}\right\|^{2} \geq \frac{\pi^{2}}{B^{2}}\|f\|^{2}=a\|f\|^{2} .
$$

On the other hand, 


$$
a\|f\|^{2} \leq\|\nabla f\|^{2}=-\int_{D} f \Delta f d x \leq\|\Delta f\|\|f\| .
$$

This implies

$$
a\|f\| \leq\|\Delta f\| \text { and } a^{2}\|f\|^{2} \leq\|\Delta f\|^{2} .
$$

Consequently, $a\|\nabla f\|^{2} \leq\|\Delta f\|^{2}$. Proof of Lemma 3.1 is completed.

In $Q_{t}$ consider the following initial boundary value problem:

$$
\begin{aligned}
& \left(u_{j}\right)_{t}+\Delta^{2} u_{j}+\Delta u_{j}+\frac{1}{2} \sum_{i=1}^{3}\left(u_{i}^{2}\right)_{x_{j}}=0, \\
& \left(u_{i}\right)_{x_{j}}=\left(u_{j}\right)_{x_{i}}, j \neq i, i, j=1, \ldots, 3 \\
& \left.u_{j}\right|_{\partial D}=\left.\frac{\partial}{\partial N} u_{j}\right|_{\partial D}=0, t>0 \\
& u_{j}(x, 0)=u_{j 0}(x), j=1, \ldots, 3, x \in D
\end{aligned}
$$

where $\frac{\partial}{\partial N}$ is an exterior normal derivative on $\partial D$.

Theorem 3.1. Let

$$
B<\pi, a=\frac{\pi^{2}}{B^{2}}, \theta=1-\frac{1}{a}>0 .
$$

Given $u_{j 0} \in H^{4}(D) \cap H_{0}^{2}(D), j=1,2,3$ such that

$$
\theta-\frac{48}{\theta a^{\frac{3}{2}}} \sum_{i=1}^{3}\left\|u_{i 0}\right\|^{2}>0
$$

Then the problem (3.3)-(3.6) has a unique strong solution

$$
\begin{aligned}
& u_{j} \in L^{\infty}\left(\mathbb{R}^{+} ; H_{0}^{2}(D)\right), \Delta^{2} u_{j} \in L^{\infty}\left(\mathbb{R}^{+} ; L^{2}(D)\right) ; \\
& u_{j t} \in L^{\infty}\left(\mathbb{R}^{+} ; L^{2}(D)\right) \cap L^{2}\left(\mathbb{R}^{+} ; H_{0}^{2}(D)\right), j=1,2,3 .
\end{aligned}
$$

Moreover, $u_{j}$ satisfies the following inequality

$$
\sum_{j=1}^{3}\left\|u_{j}\right\|^{2}(t)+\frac{\theta}{2} \int_{0}^{t}\left(\sum_{j=1}^{3}\left\|\Delta u_{j}\right\|^{2}(\tau)\right) d \tau \leq \sum_{j=1}^{3}\left\|u_{j 0}\right\|^{2},
$$




$$
\begin{aligned}
& \sum_{j=1}^{3}\left\|u_{j}\right\|^{2}(t) \leq\left[\sum_{j=1}^{3}\left\|u_{j 0}\right\|^{2}\right] \exp \left\{-\frac{a^{2} \theta t}{2}\right\} . \\
& \sum_{j=1}^{3}\left\|u_{j t}\right\|^{2}(t) \leq\left(\sum_{j=1}^{3}\left\|u_{j t}\right\|^{2}(0)\right) \exp \left\{-\frac{a^{2} \theta t}{2}\right\}, \\
& \sum_{j=1}^{3}\left\|u_{j t}\right\|^{2}(t)+\frac{\theta}{2} \int_{0}^{t}\left(\sum_{j=1}^{3}\left\|\Delta u_{j \tau}\right\|^{2}(\tau)\right) d \tau \leq \sum_{j=1}^{3}\left\|u_{j t}\right\|^{2}(0),
\end{aligned}
$$

where

$$
t>0,\left\|u_{j t}\right\|^{2}(0) \| \leq C\left(\left\|u_{0 j}\right\|_{W}\right), j=1,2,3
$$

Proof. Define the space $W=H^{4}(D) \cap H_{0}^{2}(D)$ and let $\left\{w_{i}(x), i \in \mathbb{N}\right\}$ be a countable dense set in $W$. We can construct approximate solutions to (9)-(12) in the form

$$
u_{j}^{N}(x, t)=\sum_{i=1}^{N} g_{i}^{j}(t) w_{i}(x) ; j=1,2,3
$$

Unknown functions $g_{i}^{j}(t)$ satisfy the following initial problems:

$$
\begin{aligned}
& \frac{d}{d t}\left(u_{j}^{N}, w_{j}\right)(t)+\left(\Delta u_{j}^{N}, \Delta w_{j}\right)(t)-\left(\nabla u_{j}^{N}, \nabla w_{j}\right)(t)-\frac{1}{2} \sum_{i=1}^{3}\left(\left(u_{i}^{N}\right)^{2},\left(w_{j}\right)_{x_{j}}\right)(t)=0, \\
& g_{i}^{j}(0)=g_{i 0}^{j}, \mathrm{~J}=1,2,3 ; i=1,2, \ldots .
\end{aligned}
$$

By Caratheodory's existence theorem, [20], Theorems 1.2 of Chapter 1, there exist solutions of (19)-(20) at least locally in $t$. All the estimates we will prove will be done on smooth solutions of (9)-(12). Naturally, the same estimates are true also for approximate solutions $u_{j}^{N}$.

Estimate I Multiply (9) by $2 u_{j}$ to obtain

$$
\frac{d}{d t}\left\|u_{j}\right\|^{2}(t)+2\left\|\Delta u_{j}\right\|^{2}(t)-2\left\|\nabla u_{j}\right\|^{2}(t)-\sum_{i=1}^{3}\left(u_{i}^{2},\left(u_{j}\right)_{x_{j}}\right)(t)=0, j=1,2,3
$$

Since $a\left\|\nabla u_{j}\right\|^{2}(t) \leq\left\|\Delta u_{j}\right\|^{2}(t)$, taking into account (13), we get

$$
\frac{d}{d t}\left\|u_{j}\right\|^{2}(t)+\theta\left\|\Delta u_{j}\right\|^{2}(t)+\theta\left\|\Delta u_{j}\right\|^{2}(t)-\sum_{i=1}^{3}\left(u_{i}^{2},\left(u_{j}\right)_{x_{j}}\right)(t) \leq 0
$$

Making use of Lemmas 2.3, 3.1, we estimate 


$$
\begin{aligned}
I & =\sum_{i=1}^{3}\left(u_{i}^{2},\left(u_{j}\right)_{x_{j}}\right) \leq \sum_{i=1}^{3}\left\|u_{i}\right\|\left\|u_{i}\right\|_{L^{4}(D)}\left\|\left(u_{j}\right)_{x_{j}}\right\|_{L^{4}(D)} \\
& \leq \sum_{i=1}^{3} 2\left\|u_{i}\right\|\left\|u_{i}\right\|^{\frac{1}{4}}\left\|\nabla u_{i}\right\|^{\frac{3}{4}}\left\|\nabla u_{j}\right\|^{\frac{1}{4}}\left\|\Delta u_{j}\right\|^{\frac{3}{4}} \\
& \leq 2 \sum_{i=1}^{3} \frac{1}{a^{1 / 4}}\left\|u_{i}\right\|\left\|\nabla u_{i}\right\|\left\|\Delta u_{j}\right\| \leq \epsilon\left\|\Delta u_{j}\right\|^{2}+\frac{1}{\epsilon}\left(\sum_{i=1}^{3} \frac{1}{a^{1 / 4}}\left\|u_{i}\right\|\left\|\nabla u_{i}\right\|\right)^{2} \\
& \leq \epsilon\left\|\Delta u_{j}\right\|^{2}+\frac{4}{a^{1 / 2}} \sum_{i=1}^{3}\left\|u_{i}\right\|^{2}\left\|\nabla u_{i}\right\|^{2} \\
& \leq \epsilon\left\|\Delta u_{j}\right\|^{2}+\frac{4}{a^{3 / 2} \epsilon} \sum_{i=1}^{3}\left\|u_{i}\right\|^{2}\left\|\nabla u_{i}\right\|^{2},
\end{aligned}
$$

where $\epsilon$ is an arbitrary positive number. Taking $2 \epsilon=\theta$, substituting $I$ into (22) and summing up over $j=1,2,3$, we get

$$
\frac{d}{d t} \sum_{j=1}^{3}\left\|u_{j}\right\|^{2}(t)+\frac{\theta}{2} \sum_{j=1}^{3}\left\|\Delta u_{j}\right\|^{2}(t)+\left[\theta-\frac{24}{\theta a^{3 / 2}} \sum_{i=1}^{3}\left\|u_{i}\right\|^{2}(t)\right] \sum_{j=1}^{3}\left\|\Delta u_{j}\right\|^{2}(t) \leq 0 .
$$

Condition (14), positivity of the second term in (23) and standard arguments guarantee that

$$
\left[\theta-\frac{24}{\theta a^{3 / 2}} \sum_{i=1}^{3}\left\|u_{i}\right\|^{2}(t)\right]>0, t>0
$$

This transforms (23) into the following inequality:

$$
\frac{d}{d t} \sum_{j=1}^{3}\left\|u_{j}\right\|^{2}(t)+\frac{\theta}{2} \sum_{j=1}^{3}\left\|\Delta u_{j}\right\|^{2}(t) \leq 0
$$

Integrating (25), we obtain

$$
\sum_{j=1}^{3}\left\|u_{j}\right\|^{2}\left(t+\int_{0}^{t} \frac{\theta}{2} \sum_{j=1}^{3}\left\|\Delta u_{j}\right\|^{2}(\tau) d \tau \leq \sum_{j=1}^{3}\left\|u_{j 0}\right\|^{2}\right.
$$

On the other hand, Lemma 3.1 allows us to rewrite (25) in the form

$$
\frac{d}{d t} \sum_{j=1}^{3}\left\|u_{j}\right\|^{2}(t)+\frac{a^{2} \theta}{2} \sum_{j=1}^{3}\left\|u_{j}\right\|^{2}(t) \leq 0
$$

From this, (16) follows.

Estimate II Differentiate (9) with respect to $t$, then multiply the results respectively by $2\left(u_{j}\right)_{t}$ to get 


$$
\frac{d}{d t}\left\|u_{j t}\right\|^{2}(t)+2\left\|\Delta u_{j t}\right\|^{2}(t)-2\left\|\nabla u_{j t}\right\|^{2}(t)=2 \sum_{i=1}^{3}\left(u_{i} u_{i t},\left(u_{j}\right)_{x_{j t}}\right)(t), j=1,2,3 .
$$

Making use of Lemmas 2.4 and 3.1, we estimate

$$
\begin{aligned}
I & =2 \sum_{i=1}^{3}\left(u_{i} u_{i t},\left(u_{j}\right)_{x_{j t}}\right)(t) \leq 2 \sum_{i=1}^{3}\left\|u_{i}\right\|(t)\left\|u_{i t}\right\|_{L^{4}(D)}(t)\left\|\nabla u_{j t}\right\|_{L^{4}(D)}(t) \\
& \leq 4 \sum_{i=1}^{3}\left\|u_{i}\right\|\left\|u_{i t}\right\|^{\frac{1}{4}}\left\|\nabla u_{i t}\right\|^{\frac{3}{4}}\left\|\nabla u_{i t}\right\|^{\frac{1}{4}}\left\|\Delta u_{i t}\right\|^{\frac{3}{4}} \\
& \leq 4 \sum_{i=1}^{3} \frac{1}{a^{1 / 4}}\left\|u_{i}\right\|\left\|\nabla u_{i t}\right\|\left\|\Delta u_{i t}\right\| \leq \epsilon\left\|\Delta u_{i t}\right\|^{2}+\frac{4}{\epsilon}\left(\sum_{i=1}^{3} \frac{1}{a^{1 / 4}}\left\|u_{i}\right\|\left\|\nabla u_{i t}\right\|\right)^{2} \\
& \leq \epsilon\left\|\Delta u_{i t}\right\|^{2}+\frac{16}{a^{1 / 2} \epsilon} \sum_{i=1}^{3}\left\|u_{i}\right\|^{2}\left\|\nabla u_{i t}\right\|^{2} \\
& \leq \epsilon\left\|\Delta u_{i t}\right\|^{2}+\frac{16}{a^{3 / 2}} \sum_{i=1}^{3}\left\|u_{i}\right\|^{2}\left\|\Delta u_{i t}\right\|^{2} .
\end{aligned}
$$

Taking $2 \epsilon=\theta$, substituting $I$ into (28) and summing up over $j=1,2,3$, we get

$$
\frac{d}{d t} \sum_{j=1}^{3}\left\|u_{j t}\right\|^{2}(t)+\frac{\theta}{2} \sum_{j=1}^{3}\left\|\Delta u_{j t}\right\|^{2}(t)+\left[\theta-\frac{48}{\theta a^{3 / 2}} \sum_{i=1}^{3}\left\|u_{i}\right\|^{2}(t)\right] \sum_{j=1}^{3}\left\|\Delta u_{j t}\right\|^{2}(t) \leq 0 .
$$

Taking into account (14), rewrite (29) in the form

$$
\frac{d}{d t} \sum_{j=1}^{3}\left\|u_{j t}\right\|^{2}(t)+\frac{\theta}{2} \sum_{j=1}^{3}\left\|\Delta u_{j t}\right\|^{2}(t) \leq 0 .
$$

This implies

$$
\sum_{j=1}^{3}\left\|u_{j t}\right\|^{2}(t)+\frac{\theta}{2} \int_{0}^{t}\left(\sum_{j=1}^{3}\left\|\Delta u_{j \tau}\right\|^{2}(\tau) d \tau \leq \sum_{j=1}^{3}\left\|u_{j t}\right\|^{2}(0),\right.
$$

where $\left\|u_{j t}\right\|^{2}(0) \leq C\left(\left\|u_{0 j}\right\|_{W}\right)$. Making use of Lemma 3.1, rewrite (30) as

$$
\frac{d}{d t} \sum_{j=1}^{3}\left\|u_{j t}\right\|^{2}(t)+\frac{a^{2} \theta}{2} \sum_{j=1}^{3}\left\|u_{j t}\right\|^{2}(t) \leq 0 .
$$

Integrating this, we find 


$$
\sum_{j=1}^{3}\left\|u_{j t}\right\|^{2}(t) \leq\left(\sum_{j=1}^{3}\left\|u_{j t}\right\|^{2}(0)\right) \exp \left\{-\frac{a^{2} \theta}{2} t\right\} .
$$

Estimates (3.20) and (3.25) imply that $u_{j} \in L^{\infty}\left(\mathbb{R}^{+} ; H_{0}^{2}(D)\right)$,

$$
u_{j t} \in L^{\infty}\left(\mathbb{R}^{+} ; L^{2}(D)\right) \cap L^{2}\left(\mathbb{R}^{+} ; H_{0}^{2}(D)\right), j=1,2,3 .
$$

These inequalities guarantee the existence of strong solutions $\left\{u_{j}(x, t)\right\}$ to (9)-(12) satisfying the following identity:

$$
\left.\left(\left(u_{j}\right)_{t}, \phi\right)(t)+\left(\Delta u_{j}, \Delta \phi\right)(t)+\left(\Delta u_{j}, \phi\right)(t)-\frac{1}{2} \sum_{i=1}^{3}\left(u_{i}\right)^{2}, \phi_{x_{j}}\right)(t)=0, t>0,
$$

where $\phi(x, y)$ is an arbitrary function from $H_{0}^{2}(D)$.

We can rewrite (34) in the form

$$
\left(\Delta u_{j}, \Delta \phi\right)(t)=-\left(\left[\left(u_{j}\right)_{t}+\Delta u_{j}+\sum_{i=1}^{3} u_{i}\left(u_{i}\right)_{x_{j}}\right], \phi\right)(t)
$$

Since $u_{j} \in L^{\infty}\left(\mathbb{R}^{+} ; H_{0}^{2}(D)\right)$, it follows from here and estimates (26), (31) that

$$
\left[\left(u_{j}\right)_{t}+\Delta u_{j}+\sum_{i=1}^{3} u_{i}\left(u_{i}\right)_{x_{j}}\right] \in L^{\infty}\left(\mathbb{R}^{+} ; L^{2}(D)\right), j=1,2,3,
$$

hence

$$
\Delta^{2} u_{j} \in L^{\infty}\left(\mathbb{R}^{+} ; L^{2}(D)\right), j=1,2,3
$$

This proves the existence part of Theorem 3.1.

Lemma 3.2. The strong solutions of (9)-(12) is unique. system:

Proof. Let $u_{j}$ and $v_{j}, j=1,2,3$, be two distinct solutions to (9)-(12). Denoting $w=u_{j}-v_{j}$, we come to the following

$$
\begin{aligned}
& \frac{d}{d t}\left\|w_{j}\right\|^{2}(t)+2\left\|\Delta w_{j}\right\|^{2}(t)-2\left\|\nabla w_{j}\right\|^{2}(t)=\sum_{i=1}^{4}\left(\left\{u_{i}+v_{i}\right\} w_{i},\left(w_{j}\right)_{x_{j}}\right)(t), \\
& \left(w_{i}\right)_{x_{j}}=\left(w_{i}\right)_{x_{j}}, i \neq j, \\
& \left.w_{j}\right|_{\partial D}=\left.\frac{\partial}{\partial N} w_{j}\right|_{\partial D}=0, t>0, \\
& w_{j}(x, 0)=0, j=1,2,3 .
\end{aligned}
$$

Making use of Lemmas 2.3, 3.1, we estimate 


$$
\begin{aligned}
I & =\left(\left\{u_{i}+v_{i}\right\} w_{i},\left(w_{j}\right)_{x_{j}}\right) \leq\left\|\left(w_{j}\right)_{x_{j}}\right\|_{L^{4}(D)}\left\|w_{i}\right\|\left\|u_{i}+v_{i}\right\|_{L^{4}(D)} \\
& \leq 2\left\|w_{i}\right\|\left\|\left(w_{j}\right)_{x_{j}}\right\|^{\frac{1}{4}}\left\|\nabla\left(w_{j}\right)_{x_{j}}\right\|^{\frac{3}{4}}\left\|u_{i}+v_{i}\right\|^{\frac{1}{4}}\left\|\nabla\left(u_{i}+v_{i}\right)\right\|^{\frac{3}{4}} \\
& \leq \frac{2}{a^{1 / 4}}\left\|w_{i}\right\|\left\|\Delta\left(w_{j}\right)\right\|\left\|\nabla\left(u_{i}+v_{i}\right)\right\| \\
& \leq \epsilon\left\|\Delta\left(w_{j}\right)\right\|^{2}+\frac{1}{\epsilon a^{3 / 2}}\left\|w_{i}\right\|^{2}\left\|\Delta\left(u_{i}+v_{i}\right)\right\|^{2} .
\end{aligned}
$$

Substituting $I$ into (35), taking $2 \epsilon=\theta$ and summing over $j=1,2,3$, we get

$$
\frac{d}{d t} \sum_{j=1}^{3}\left\|w_{j}\right\|^{2}(t)+\theta \sum_{j=1}^{3}\left\|\Delta w_{j}\right\|^{2}(t) \leq C\left(\sum_{i=1}^{3}\left\{\left\|\Delta u_{i}\right\|^{2}(t)+\left\|\Delta v_{i}\right\|^{2}(t)\right\} \sum_{j=1}^{3}\left\|w_{j}\right\|^{2}(t)\right) .
$$

Due to (26), $\left\|\Delta u_{i}\right\|^{2}(t)+\left\|\Delta v_{i}\right\|^{2}(t) \in L^{1}\left(\mathbb{R}^{+}\right), i=1,2,3$.

Applying Lemma 2.2, we obtain that

$$
\left\|w_{j}\right\|(t) \equiv 0, t>0, j=1,2,3
$$

This proves Lemma 3.2 and consequently Theorem 3.1.

Remark 3.1. We can define other grooves:

$$
\begin{aligned}
& D_{x_{3}}=\left\{x=\left(x_{1}, x_{2}, x_{3}\right) ; x_{1} \in \mathbb{R}^{1}, x_{2} \in \mathbb{R}^{+}, x_{3} \in\left(0, L_{3}\right),\left(L_{3}>0\right),\right\} \\
& D_{x_{1}}=\left\{x=\left(x_{1}, x_{2}, x_{3}\right) ; x_{1} \in\left(0, L_{1}\right),\left(L_{1}>0\right), x_{2} \in \mathbb{R}^{1}, x_{3} \in \mathbb{R}^{+}\right\}
\end{aligned}
$$

and obtain results similar to ones of Theorem 3.1.

\section{Possible applications of theorem 3.1}

Since by [2], the Kuramoto-Sivashinsky equation describes instabilities in laminar flame fronts, it can be used to create mathematical models of wildfires. On the other hand, the Kuramoto-Sivashinsky equation describes also the dynamics of turbulent phase waves and flow of liquid films on an inclined plane [4]. This can be used to create mathematical models for the stability of processes in river flows.

\section{Data availability statement}

I declare that all the data that have been used in my manuscript are mathematical theorems published in open mathematical journals and presented in References. 


\section{Conclusions}

In this work, we studied the stability of global solutions for initial boundary value problems to the threedimensional Kuramoto-Sivashinsky system (1) posed on unbounded grooves. We defined a set of admissible domains which eliminate destabilizing effects of terms $\Delta u_{j}$ by dissipativity of $\Delta^{2} u_{j}$. Since these problems do not admit the first a priori estimate independent of $t$ and solutions, in order to prove the existence of global solutions, we put conditions connecting geometrical properties of domains with initial data. We proved the existence and uniqueness of a strong solution as well as exponential decay of $L^{2}$-norms.

\section{Acknowledgments}

The author appreciated very much concrete and useful comments of his reviewers as well as Journal Coordinator Wendy Zhang for his support and help during the complicated submission process.

\section{Conflict of interests}

The author declares that there is no conflict of interest regarding the publication of this paper.

\section{References}

[1] Kuramoto Y, Tsuzuki T. On the formation of dissipative structures in reaction-diffusion systems. Progress of Theoretical Physics. 1975; 54(3): 687-699.

[2] Sivashinsky GI. Nonlinear analysis of hydrodynamic instability in laminar flames-1. Derivation of basic equations. Acta Astronauica. 1977; 4(11-12): 1177-1206.

[3] Cross MC. Pattern formation outside of equilibrium. Review of Modern Physics. 1993; 65(3): 851-1086.

[4] Sivashinsky GI, Michelson D. On irregular wavy flow of a liquid film down a vertical plane. Progress of Theoretical Physics. 1980; 63(6): 2112-2114.

[5] Biagioni HA, Bona JL, Iorio Jr. RJ, Scialom M. On the Korteweg-de Vries-Kuramoto-Sivashinsky equation. $A d v$. Differential Equations. 1996; 1(1): 1-20.

[6] Cousin AT, Larkin NA. Kuramoto-Sivashinsky equation in domains with moving boundaries. Portugaliae Mathematica. 2002; 59(3): 335-349.

[7] Feng BF, Malomed BA, Kawahara T. Stable periodic waves in coupled sivashinsky-korteweg-de vries equations. Cornell University; 2002.

[8] Li J, Zhang BY, Zhang ZX. A non homogeneous boundary value problem for the Kuramoto-Sivashinsky equation in a finite interval. ESAIM Control Optimisation and Calculus of Variations. 2020; 26: 43. Available from: https:// doi.org/10.1051/cocv/2019027.

[9] Biagioni HA, Gramchev T. Multidimensional Kuramoto-Sivashinsky type equations: Singular initial data and analytic regularity. Matemática Contemporanea.1998; 15: 21-42.

[10] Benachour S, Kukavica I, Rusin W, Ziane M. Anisotropic estimates for the two dimensional Kuramoto-Sivashinsky equation. Journal of Dynamics and Differential Equations. 2014; 26: 461-476. Available from: https://doi. org/10.1007/s10884-014-9372-3.

[11] Larkin NA. Korteweg-de Vries and Kuramoto-Sivashinsky equations in bounded domains. Journal of Mathematical Analysis and Applications. 2004; 297(1): 169-185.

[12] Molinet L. Local dissipativity in $\mathrm{L}^{2}$ for the Kuramoto-Sivashinsky equation in spatial dimension 2. Journal of Dynamics and Differential Equations. 2000; 12: 533-556.

[13] Sell GR, Taboada M. Local dissipativity and attractors for the Kuramoto-Sivashinsky equation in thin 2D domains. Nonlinear Analysis. 1992; 18(7): 671-687.

[14] Nicolaenko B, Scheurer B, Temam R. Some global dynamical properties of the Kuramoto-Sivashinsky equations: nonlinear stability and attractors. Physica D: Nonlinear Phenomena. 1985; 16(2): 155-183.

[15] Temam R. Infinite-dimensional dynamical systems in mechanics and physics. Berlin-Heidelberg: Springer; 1988. 
[16] Adams RA, Fournier JF. Sobolev spaces. Oxford OX5 IGB UK: Elsevier Science Ltd; 2003.

[17] Steklov AV. The problem of cooling of an heterogeneous rigid rod. Communications of the Kharkov Mathematical Society. 1896; 2(5): 136-181.

[18] Larkin NA, Padilha MV. Decay of solutions for 2D Navier-Stokes equations posed on Lipschitz and smooth bounded and unbounded domains. Journal of Differential Equations. 2019; 266(11): 7545-7568.

[19] Temam R. Navier-stokes equations. Theory and numerical analysis. North-Holland, Amsterdam: Noth-Holland; 1977.

[20] Coddington E, Levinson N. Theory of ordinary differential equations. New York: MacGraw-Hill; 1955. 